\title{
AFI: UN APORTE A LA CALIDAD
}

\author{
Fernando Rojas*
}

Andrés Bernasconi*

* Oficina de Análisis Institucional de la Universidad Andrés Bello. 


\section{RESUMEN}

Con base en la información públicamente disponible del Ministerio de Educación sobre la distribución del Aporte Fiscal Indirecto (AFI), la revisión de la ley y sus modificaciones, y antecedentes más detallados sobre la distribución del AFI del año 1998, el presente artículo realiza una evaluación empírica del desempeño del AFI entre 1990 y 2002.

En el análisis se comprueba la concentración del aporte en universidades -en desmedro de los institutos profesionales y centros de formación técnica-, en instituciones del Consejo de Rectores, y en alumnos de colegios privados, de la región metropolitana y de sexo masculino. Como factores explicativos de tales patrones de distribución se proponen la decreciente movilidad interregional de los alumnos, la diversa capacidad de las regiones de producir buenos puntajes, y la relación que existe entre resultados de la PAA y el nivel socioeconómico y sexo del alumno. Se comprueba, además, la estrecha relación que existe entre AFI y prestigio institucional, así como la creciente participación de las universidades privadas en el aporte.

El análisis sugiere que la incorporación de las notas de enseñanza media al cálculo del AFI beneficiaría a las postulantes mujeres y a las universidades del Consejo Rectores, sin modificar la actual composición socioeconómica de los alumnos receptores de AFI.

Se subraya, por último, la importancia del AFI como indicador de calidad institucional, y la necesidad de mejorar el nivel de información sobre sus resultados, para así contribuir a guiar el desarrollo de la educación superior hacia comportamientos institucionales que enfaticen la orientación al usuario y la calidad de servicios y productos como factores clave en la competitividad.

\section{ABSTRACT}

Based on publicly available data from the Ministry of Education on the distribution of Indirect Public Funding (IPF), a review of the law and its amendments, and more detailed data on the distribution of IPF for the year 1998, we present an empirical assessment of the performance of IPF between 1990 and 2002.

The analysis shows a concentration of this form of funding upon universities -as compared to professional institutes and technical training centers-institutions who are members of the Council of Rectors, and male students from private schools in the metropolitan region. Explanatory factors are proposed in connection to decreasing interregional mobility of students, the varying capacity of the regions to produce good scores, and the relationship between the Scholastic Aptitude Test and the sex and SES of the student. The close relationship between participation in IPF and institutional prestige is also documented, as is the growing share of private universities in IPF.

Our analysis suggests that adding high school grades to the formula for calculating IPF would benefit female applicants and universities represented in the Council of Rectors, without altering the current socio-economic makeup of recipients of IPF.

Lastly, the importance of IPF as indicator of institutional quality is underscored, together with the need to improve the availability of data on its distribution, as a way to steer the development of higher education towards user-oriented institutional behavior orientation and quality of services and outcomes as key elements for competitiveness. 


\section{AFI: UN APORTE A LA CALIDAD}

\section{INTRODUCCIÓN}

En momentos en que, como coletazos de la discusión sobre las pruebas de admisión a la Universidad, han surgido en el debate de políticas de educación superior voces partidarias de revisar el Aporte Fiscal Indirecto (AFI), el presente artículo busca contribuir a una discusión informada del tema, ofreciendo una evaluación empírica del desempeño de dicho mecanismo de financiamiento y estímulo a la calidad entre 1990 y 2002. Se analiza primero la relación entre AFI y prestigio institucional, la evolución de los montos de financiamiento involucrados en este aporte, y los factores que atentan contra la competencia que dicho mecanismo busca estimular. Luego se examinan tanto el impacto que el AFI ha tenido en el comportamiento de las universidades, como las características socioeconómicas y demográficas de los alumnos que generan AFI. A continuación, se revisan los patrones de distribución de este aporte entre las diversas instituciones elegibles para recibirlo. Finalmente, el artículo evalúa a través de una simulación cuáles serían algunos de los efectos de modificar el AFI para incorporar a él las notas de enseñanza media como factor de asignación, y se concluye proponiendo algunas innovaciones que mejorarían la capacidad del AFI de alcanzar sus objetivos de identificación y premio a las carreras consideradas de calidad por los usuarios.

El AFI, en su modalidad original, fue establecido en el paquete de medidas del D.F.L. (E) $\mathrm{N}^{\circ} 4$, de 1981, y empezó a aplicarse en 1982. Este cuerpo legal modificó profundamente el sistema de educación superior chileno al diversificar su financiamiento e incorporar incentivos a la competencia entre las instituciones. El actual mecanismo de distribución del AFI se encuentra vigente desde $1989^{1}$,

1. Artículo $3^{\circ}$ DFL-4 de 1981, modificado por el artículo 20 de la Ley 18.681 de 1987 y el artículo 51 de la Ley 18.768 de 1988. 
año en que se incorporaron a él las instituciones de educación superior privadas (universidades, institutos profesionales y centros de formación técnica), y se fijó en 27.500 el número de alumnos que podían ser generadores de este aporte. Del mecanismo original se mantienen hasta hoy su carácter competitivo y la relación directa entre el nivel del aporte y los puntajes en la Prueba de Aptitud Académica (PAA).

La asignación del AFI considera los 27.500 alumnos que obtuvieron los mejores puntajes de la PAA, promedio partes verbal y matemática, y que se matricularon en alguna institución de educación superior. Los alumnos se ordenan de menor a mayor puntaje, separándolos en cinco tramos de 5.500 alumnos cada uno. El primer tramo recibe una ponderación de 1 , el segundo una de 3 , y así sucesivamente de tres en tres hasta la ponderación del quinto tramo, que alcanza a 12. Es decir, al alumno cuyo puntaje lo ubica en el tramo 5 le corresponde recibir 12 veces más AFI que a uno del primer tramo. La suma por institución de los valores así asociados a cada puntaje determina el monto anual de libre disposición que se asigna a cada una de ellas. Así, para el aporte del año 2002, la unidad de aporte fiscal indirecto (UAFI) ${ }^{2}$ correspondió a $\$ 99.103$ (US $\left.\$ 141\right)^{3}$; en consecuencia, un alumno en el tramo 5, es decir, con puntaje mayor a 723 puntos, proporcionó a la institución en que se matriculó \$1.189.237 ( US\$1.699).

Los alumnos que dan derecho a este aporte son los matriculados en el primer año de estudios, y que hayan rendido la PAA en el proceso de admisión correspondiente a ese año ${ }^{4}$. La distribución por el Estado de los aportes correspondientes a esos alumnos se hace al año siguiente al de su matrícula. En la distribución no se consideran los alumnos que ingresan por segunda vez a una misma institución, ni los alumnos que ingresan por tercera vez a una misma o diversas instituciones. En otras palabras, un alumno puede en la práctica generar AFI hasta dos veces, siempre que sea en dos instituciones distintas.

2. El valor de la UAFI se obtiene del monto presupuestario asignado para el aporte en el año respectivo, dividido por el número total de UAFI a distribuir, que corresponde aproximadamente a 170.500 -dependiendo del número de casos en el puntaje de corte de cada tramo- $y$ es igual a $5.500(1+3+6+9+12)$.

3. US\$ calculado a $\$ 700$ por unidad.

4. El requisito de haber rendido la PAA en el proceso de admisión del año en que el alumno se matricula aparecía en la versión original de la ley, pero no se reiteró en la modificación de 1987. No obstante, el Ministerio de Educación ha interpretado que el requisito sigue vigente. 


\section{APORTE A LA CALIDAD}

Originalmente, el propósito del AFI parece no haber sido sólo fomentar la competencia. En efecto, la norma del texto original del art. 3 del DFL 4, vigente entre 1981 y 1987, ponderaba con un multiplicador de 2.5 el aporte a los alumnos matriculados en Medicina y Odontología, y de 1.5 a los alumnos inscritos en Bioquímica, Ingeniería Civil, Agronomía, Ingeniería Forestal, Química y Farmacia y Medicina Veterinaria. Ello sugiere que un objetivo adicional del AFI pudo haber sido subsidiar directamente a las carreras de alto costo.

En la actualidad, el sentido inmediato del AFI es premiar a las instituciones que captan a los alumnos con mejores puntajes. Indirectamente, este aporte introduce también un incentivo para que las instituciones aumenten la oferta de cupos en aquellas carreras que captan altos puntajes, o las creen, si es que no las tienen. En cualquier caso, el premio a la institución pasa por la elección que de ella hace el postulante.

La lógica del AFI asume que el objetivo de un estudiante de pregrado es adquirir una sólida formación profesional en una carrera en particular y que, por lo tanto, se matriculará en la institución a su alcance que crea le proporcionará la mejor formación. Por otra parte, los alumnos con los mejores puntajes en el proceso de selección a la educación superior son los que presentan menores restricciones en cuanto al cumplimiento de los requisitos de ingreso de las instituciones, traduciéndose esto en una mayor capacidad de elección. Como consecuencia, se tiene que el número de alumnos de altos puntajes ingresados a cada carrera es un poderoso indicador de la calidad de esa carrera a juicio de quienes se disponen a convertirse en sus usuarios. El hecho de que a este indicador se liguen aportes fiscales de libre disponibilidad convierte al AFI en el único aporte dirigido a potenciar los programas de pregrado de calidad e incentivar el aumento de su oferta.

Técnicamente, este indicador cumple con las condiciones generales para que un indicador resulte operativo en los modelos de financiamiento a instituciones autónomas, a saber: 1) que refleje los objetivos de quien los establece, 2) que sea sencillo de entender y 
calcular, 3) que no sea susceptible de manipulación por parte de las instituciones, y 4) que muestre cierto nivel de estabilidad temporal.

La hipótesis de que el AFI está relacionado con el prestigio de una institución se comprueba al tomar los datos del último ranking de reputación publicado por la revista Qué Pasa $a^{5}$, el que se basa en una encuesta de opinión a académicos y profesionales sobre el la calidad de las universidades chilenas. Hemos correlacionado la nota por prestigio obtenida por cada universidad listada por Qué Pasa en 2001, con las UAFI que obtuvo cada institución en 2002 divididas por su matrícula total de primer año. El uso de UAFI por alumno de primer año permite corregir el efecto del tamaño de la institución en su captación del AFI, y permite ponderar por tramos a los alumnos con AFI.

Los resultados comprueban en forma empírica que a mayor nivel de prestigio de una institución, mayor es el número promedio de UAFI que aportan sus alumnos. La correlación entre ambas variables es de 0.76 (gráfico $\mathrm{N}^{\mathrm{o}} 1$, abajo y apéndice $\mathrm{N}^{\mathrm{o}} 1$, al final).

\section{Gráfico 1}

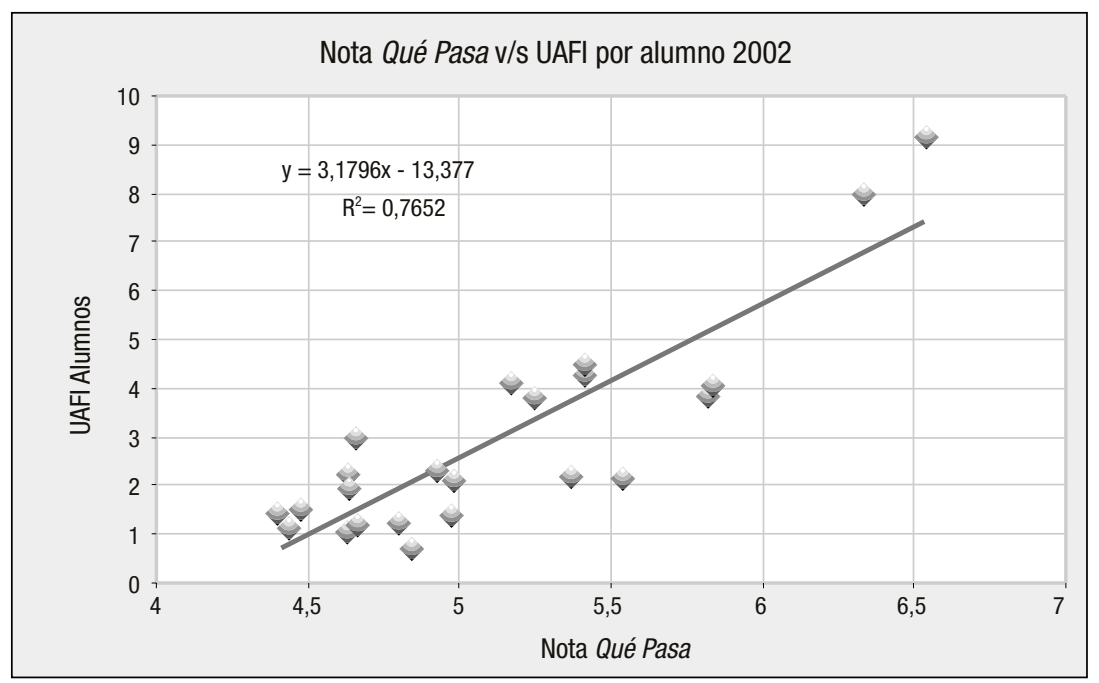

5. Revista Qué Pasa, Examen las Universidades Chilenas, Chile, diciembre de 2001. 


\section{APLICACIÓN DEL AFI ${ }^{6}$}

\subsection{Aspectos técnicos de su aplicación}

\subsubsection{Nivel de los recursos}

El poder de un incentivo para modelar la conducta de una organización en el largo plazo depende de la magnitud y permanencia de los recursos involucrados, y de la estabilidad de los indicadores utilizados, puesto que una dependencia muy fuerte de recursos inciertos o asignados con criterio variable trae consigo altos niveles de inestabilidad financiera y dificultades para planificar a largo plazo.

Podría argumentarse que los recursos del AFI son innecesarios, porque en la ausencia de ellos las instituciones de todos modos competirían por los mejores alumnos. Aunque plausible, el argumento no reconoce que el AFI también contribuye a financiar carreras de alto costo y alta selectividad. Es así como en muchas instituciones los montos recibidos por AFI son asignados por la autoridad central a las unidad académicas que los generan. Por otra parte, aunque es claro que las unidades académicas más selectivas seguirían compitiendo por los mejores alumnos si el aporte no existiera, puesto que ellos son el insumo principal para sus carreras, no es igualmente claro que carreras menos selectivas harían lo mismo. La eliminación de un incentivo al reclutamiento de buenos alumnos podría, en balance, resultar en menores opciones, o en condiciones menos ventajosas de colocación en el sistema de educación superior para los mejores alumnos.

Los datos sobre el nivel de recursos asignados al AFI muestran que éste no ha sido una prioridad política de la gestión de educación superior. Desde 1991 el aporte ha presentado pequeña pero constante disminución en términos reales, acumulando una disminución real del 11\% entre 1990 y 2002, así como una baja del 18.2\% al 7.6\% en su participación en el presupuesto público de educación superior. En comparación, el aporte fiscal directo ha experimentado en el mismo período un aumento real de más de $71 \%$ (véase gráfico № 2 ).

6. Para el análisis cuantitativo de la aplicación del AFI se toma el período de 1990 a 2002 , considerando que en el año 1989 se realizaron las últimas modificaciones al mecanismo de asignación. Para los datos sobre características de los alumnos se utiliza el año 1998 (PAA 1997), que es la más reciente base de datos a que tenemos acceso. 
Gráfico 2

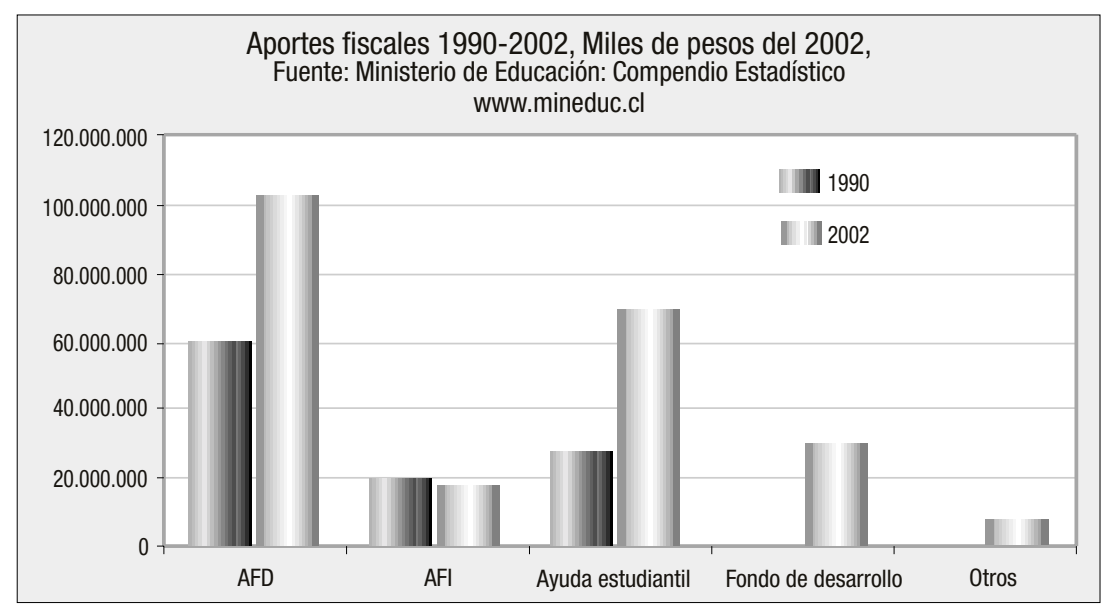

En análisis más detallado de la variación real anual y acumulada del monto asignado al AFI, se aprecia que desde 1992 existe una constante pérdida de valor real que no supera nunca el $2 \%$, pero que en el año 2000 cayó alrededor de 6\%, equivalente a más de 1.000 millones de pesos del año 2002, posiblemente porque ese año los recursos del AFI fueron utilizados como fuente de financiamiento para otros programas de educación superior (véase gráfico $\mathrm{N}^{\circ} 3$ ).

Gráfico 3

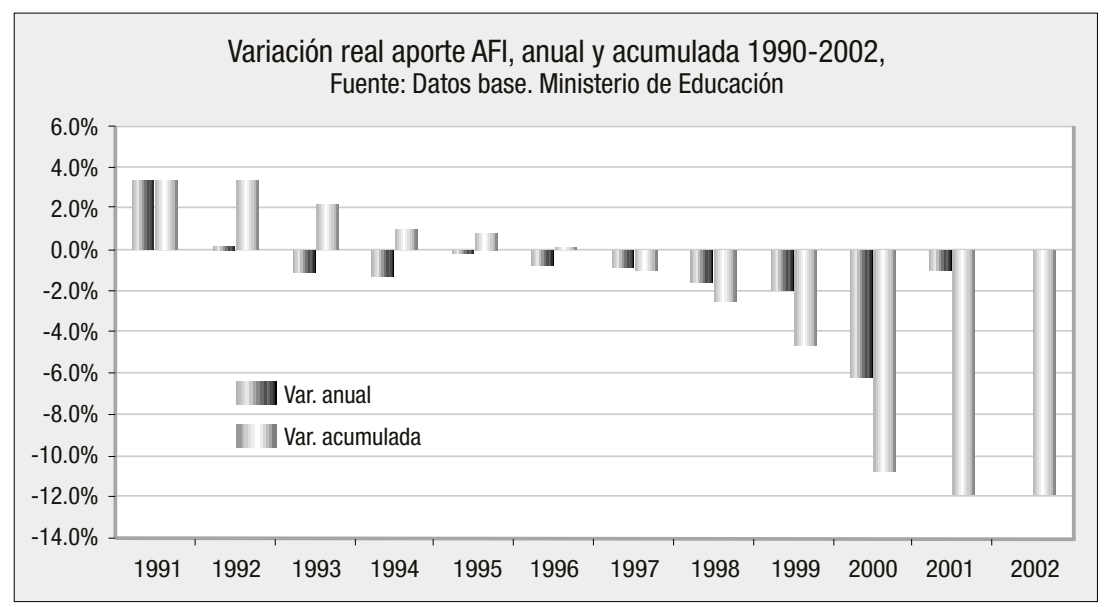




\subsubsection{Factores que atentan contra la competencia}

La función de estímulo a la competencia del AFI podría ser acentuada si se corrigieran algunas prácticas y características del sistema que dan ventajas injustificadas a algunas instituciones. Entre las situaciones referidas se encuentran:

1. El acceso exclusivo por parte de las universidades del Consejo de Rectores a las bases de datos de inscritos y resultados de la PAA da privilegios, a estas universidades sobre las demás instituciones del sistema, para identificar e intentar reclutar a los alumnos con altos puntajes.

2. La exclusividad en el acceso al crédito y a la mayoría de las becas estatales, por parte de estas mismas universidades, distorsiona el criterio de calidad como base de la elección del postulante, al introducir un factor económico en el cálculo que éste hace. Una carrera débil de una universidad con crédito estudiantil puede así matricular alumnos con mejores puntajes, y recibir más AFI, que una mejor carrera de una universidad privada.

3. Por razones culturales, las universidades son más atrayentes para los alumnos que los institutos profesionales o centros de formación técnica (CFT). Independientemente de la calidad, entonces, las universidades tienen mejores resultados en AFI que las otras instituciones. Esta distorsión se corregiría si existiesen fondos separados por tipo de institución, definidos en proporción a la participación de cada tipo de institución en la matrícula de primer año.

4. El MINEDUC entrega la información sobre AFI agregada en el nivel de la institución y no desagregada en el nivel de la carrera, con lo cual se pierde información que las instituciones podrían usar para evaluar sus resultados.

Este último punto es de particular importancia, dado que la política del gobierno ha sido no restringir la oferta de carreras, sino permitir que el mercado se autorregule. No obstante, ha ignorado la oportunidad que representa la información del AFI para que el público cuente con señales orientadoras acerca de las tendencias de selección de carreras e instituciones de los postulantes que tienen mayor rango 
de elección. Esta información mostraría, por ejemplo, la pérdida de interés en determinadas carreras en los últimos años por parte de los mejores alumnos (Ingeniería Comercial es un ejemplo), y la posición de mercado que ocupa cada institución en determinadas áreas, con lo cual las instituciones podrían reorientar su oferta a las carreras en donde tienen mejores resultados comparativos. Al igual como se compara el AFI institucional, se podría comparar el AFI en el nivel de programas.

Por lo demás, la información pública más útil para el postulante es la de carreras, cuestión que los rankings empiezan a reconocer. El MINEDUC debiera, en armonía con su política de aumentar la información y trasparencia del sistema, poner estos datos a disposición de las instituciones y del público.

\subsection{Aspectos institucionales de la aplicación del AFI 3.2.1. El AFI como "voucher"}

La competencia entre las instituciones por captar alumnos con AFI se manifiesta en muchas de ellas -especialmente privadas- en la oferta de beneficios económicos a los postulantes con buenos puntajes, tales como becas, rebajas de aranceles, créditos institucionales, etcétera. Así, el AFI es "devuelto" en beneficios al alumno que lo genera ${ }^{7}$. En este caso, a la institución le interesa reclutar al alumno menos por el aporte económico que acarrea, que por su calidad académica o el prestigio que una alta matrícula de alumnos AFI representa. Estos beneficios tienden a neutralizar el efecto distorsionador del crédito universitario y, en la práctica, transforman el AFI en una especie de voucher. Estas estrategias competitivas han sido empleadas por instituciones tradicionales y privadas, con distintos objetivos de alumnos, generando una gran cantidad de recursos para los estudiantes en complemento a los sistemas de ayuda estudiantil.

\subsubsection{Incidencia del AFI en los requisitos de ingreso}

Por otro lado, el AFI se ha convertido en una causa de la universalidad de la PAA como factor de ingreso a las universidades. No se debe olvidar que el sistema privado actualmente abarca a más del 52\% de la

7. En ocasiones con creces. Por ejemplo, un descuento del $25 \%$ de arancel para alumnos en tramo 5, en una carrera de 1.9 millones anuales, por cinco o seis años, corresponde al doble del AFI generado por alumno. 
matrícula de educación superior y no existe ninguna obligación de utilizar la PAA como mecanismo de selección fuera del ámbito de las universidades del Consejo de Rectores.

Esto ha llevado, como era de esperar, a un aumento de la ponderación que tiene la PAA (verbal y matemáticas) dentro del puntaje de ingreso a cada carrera: a mayor la ponderación de la PAA verbal y matemática, mayor será la probabilidad de matricular alumnos que generen AFI (véase cuadro $\mathrm{N}^{0} 1$ ). Esto va en desmedro de la ponderación de las pruebas específicas y las notas de enseñanza media ${ }^{8}$. Las distorsiones también alcanzan a la enseñanza media, donde observadores advierten una paulatina inflación en las notas, como una forma de mejorar el puntaje ponderado de sus egresados

Cuadro $\mathrm{N}^{0} 1$

\begin{tabular}{|lcc|}
\hline \multicolumn{3}{|c|}{$\begin{array}{c}\text { Promedio de porcentaje ponderación por } \\
\text { carrera del puntaje en la PAA (verbal y matemáticas) }\end{array}$} \\
Sector & 1990 & 2001 \\
\hline Universidades con aporte fiscal & $54.4 \%$ & $59.4 \%$ \\
Universidades privadas & $42.2 \%$ & $55.9 \%$ \\
\hline
\end{tabular}

Estos cambios han sido más fuertes y han tenido una mayor variabilidad en el sistema privado ${ }^{9}$. Al interior de cada sector existen instituciones que han mantenido e incluso han bajado la ponderación a la PAA, pero el promedio se ha movido al alza.

El hecho de que algunas instituciones usen sus requisitos de ingreso para maximizar el incentivo es un efecto esperable de cualquier sistema de incentivos (piénsese en el 5\% del aporte fiscal directo, por ejemplo, que el Estado entrega según indicadores de desempeño). Lo importante es anticipar estas formas de comportamiento estratégico y tomarlas en cuenta en el diseño de las políticas.

8. Para el caso de las instituciones del Consejo de Rectores, el mínimo de ponderación de las notas es del $20 \%$.

9. Entre una ponderación de $100 \%$ de la PAA en la U. Mayor, hasta un $0 \%$ en la U. de las Américas. En el sector tradicional el cambio ha sido más moderado, llegado a un máximo del $70 \%$ de ponderación de la PAA verbal y matemática en la U. Católica de Valparaíso, por ejemplo. 


\subsubsection{Indicador de posicionamiento de mercado}

Un resultado del AFI, probablemente no esperado pero razonable y apropiado, ha sido que las instituciones usen el resultado de la asignación del aporte como un indicador de posicionamiento de mercado. De hecho, es difícil encontrar un indicador de demanda más parsimonioso que el AFI, siempre que sea bien usado.

Lamentablemente, el análisis apresurado que hacen las instituciones y los medios conduce a errores de interpretación, como omitir la información que entregan los tramos de puntaje (falla que el uso de la UAFI como unidad de medida corrige) o, más importante, no corregir por el tamaño institucional (por ejemplo, dividiendo las UAFI por matrícula total de primer año), puesto que instituciones con más alumnos tienen una mayor probabilidad de captar un mayor número absoluto de alumnos con AFI.

\subsection{Perfil de los alumnos con AFI}

\subsubsection{Colegio de procedencia}

Según los datos del AFI 1998, el 43\% de los alumnos que lo obtiene proviene de colegios privados, el $29 \%$ de colegios subvencionados y el $28 \%$ de colegios municipales, lo que está en relación directa con el rendimiento de estos colegios en la PAA.

\section{Cuadro $\mathrm{N}^{0} 2$}

\begin{tabular}{|lcc|}
\hline \multicolumn{3}{|c|}{ Número de alumnos con AFI en 1998, } \\
según dependencia del colegio de procedencia \\
$\begin{array}{l}\text { Dependencia colegio del } \\
\text { alumno }\end{array}$ & $\begin{array}{c}\text { Número de alumnos } \\
\text { con AFI, 1998 }\end{array}$ & $\begin{array}{c}\% \text { participación, } \\
\text { por dependencia }\end{array}$ \\
\hline Particular pagado & 11.822 & $42.9 \%$ \\
Particular subvencionado & 8.036 & $29.1 \%$ \\
Municipal & 7.690 & $27.9 \%$ \\
Sin información & 24 & $0.1 \%$ \\
Total & 27.572 & $100.0 \%$ \\
\hline
\end{tabular}


Esta situación se profundiza más a medida que aumenta el puntaje. Es así como alumnos de colegios particulares pagados representan el 60\% del tramo 5 (5.500 mejores puntajes), en cambio los colegios subvencionados y municipales muestran una curva inversa, con una mayor participación en los tramos bajos y descendiendo en los tramos de alto puntaje (gráfico $\mathrm{N}^{\circ} 4$ abajo, y apéndice $\mathrm{N}^{\mathrm{o}} 2$, al final).

Gráfico 4

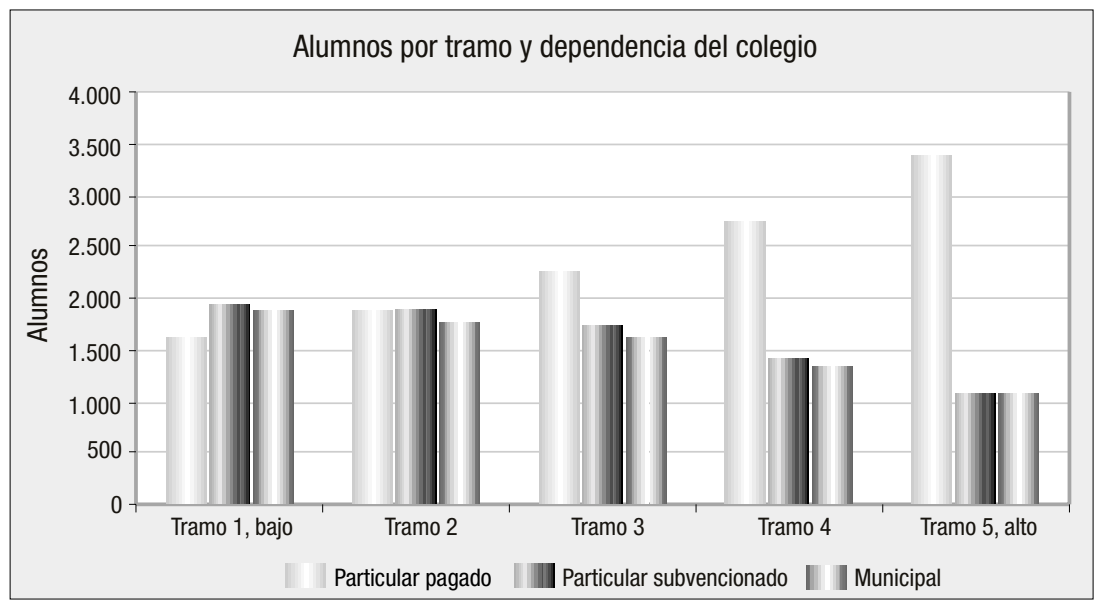

Estas cifras, que sugieren la concentración de los recursos del AFI en los alumnos más ricos, se esgrimen para subrayar el carácter socialmente regresivo del AFI. Esta posición olvida que el AFI no tiene por objetivo corregir la desigualdad social de la educación. Un instrumento de política no se juzga por su capacidad de alcanzar los objetivos que no persigue. El problema de la equidad tiene sus instrumentos de política, destinados a dar una mejor educación a los niños y jóvenes de los colegios municipalizados y subvencionados. El fomento de la calidad en la educación, por su parte, tiene los suyos, orientados a identificar y, en este caso, premiar la calidad. 


\subsubsection{Sexo}

El 55\% de los alumnos con AFI son hombres, a pesar de que los varones representan el 48\% de los inscritos en la PAA (véase apéndice $\mathrm{N}^{\circ} 3$, al final). Los hombres con AFI obtienen un puntaje promedio 8.5 puntos mayor al de las mujeres, pero esto contrasta con la diferencia en el promedio de notas de enseñanza media, donde el puntaje promedio de los hombres es menor en 48.2 puntos al de las mujeres. Si se comparan puntajes ponderados ${ }^{10}$, que incorporan las notas de enseñanza media, las mujeres superan a los hombres en 2.5 puntos.

\subsubsection{Año egreso enseñanza media.}

El $60 \%$ de los alumnos con AFI egresaron el año en que rinden la PAA; un 23\% corresponde al año anterior, y sólo un $5.8 \%$ es la participación de promociones de más de dos años de egreso (Apéndice $\mathrm{N}^{0} 4$, al final). Se advierte aquí el efecto de la regla del AFI que sólo permite a los alumnos recibir dos veces AFI en distintas instituciones. Si se examina la distribución de alumnos según tramo de AFI 1998 y año de egreso, se observa que los egresados del año se concentran en los tramos altos, partiendo con un $68.5 \%$ del tramo 5 y bajando a un $55 \%$ del tramo 1 (menores puntajes). Para las restantes promociones se advierte la situación inversa.

Gráfico 5

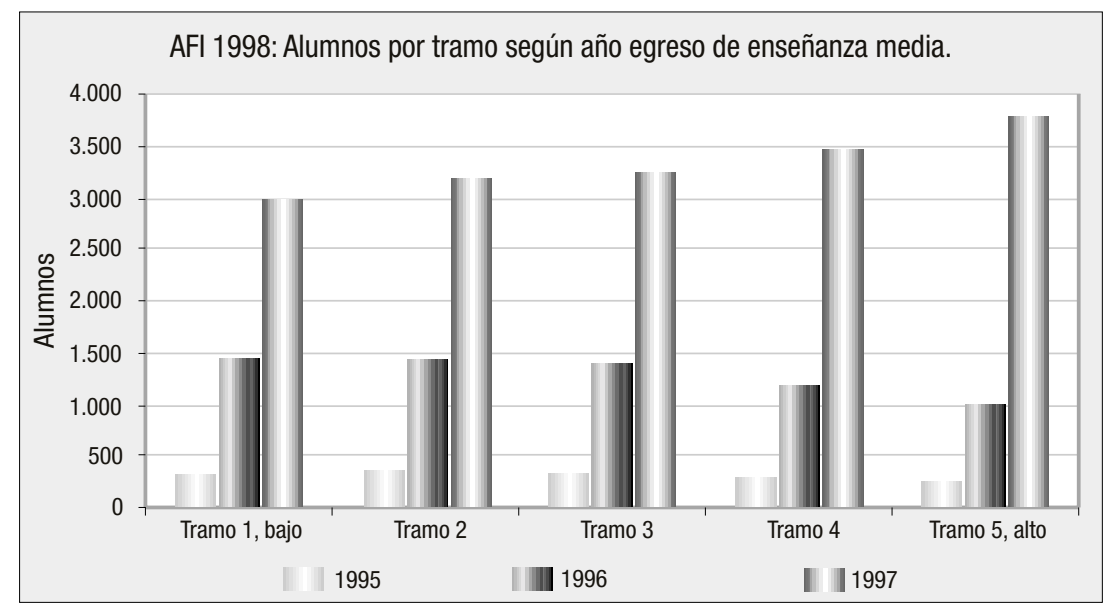

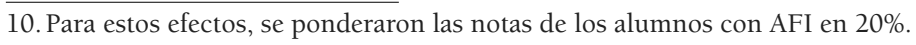




\subsubsection{Nivel educacional del padre}

Como es de esperar, a mayor nivel educacional del padre del alumno, mejor será su puntaje en la PAA y, por lo tanto, su tramo de AFI. Los alumnos con AFI de padres con educación superior completa ocupan un tramo promedio de 3.4 , en cambio los alumnos con AFI de padres con educación básica completa tienen un tramo promedio de 2.5.

Gráfico 6

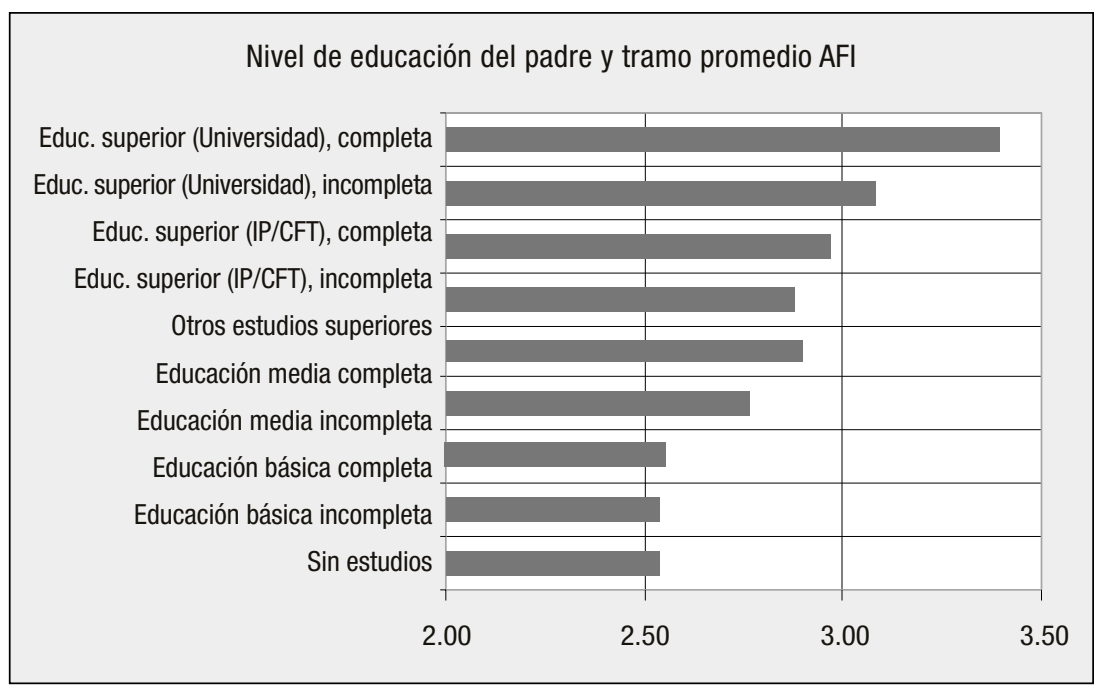

3.3.5. Procedencia geográfica del alumno con AFI y capacidad de retención de las regiones

Los alumnos con AFI provienen principalmente de tres regiones: $51 \%$ de la Región Metropolitana, 11.5\% de la Quinta Región y 11\% de la Octava Región. El resto de las regiones aporta cada una menos del 5\% de los 27.500 mejores puntajes (véase apéndice $N^{\circ}$ 5, al final).

La tasa de retención, calculada como la proporción de los alumnos con AFI de la región que se matriculan en la misma región, muestra que el $76 \%$ de los alumnos con AFI no se desplaza de su región de origen, siendo las regiones con mayor retención la Metropolitana (91.3\%), Quinta (84.2\%), Octava (81.3\%) y Novena (69.8\%). 
Existen regiones que no sólo han logrado retener a sus buenos puntajes sino además captar los de las otras. Es así como la Región Metropolitana atrae a 2.213 alumnos con AFI de otras regiones, la Quinta Región a 2.013, seguidas de lejos por la Octava, con 754, y la Segunda, con 428.

De las regiones Quinta, Sexta y Séptima en conjunto proviene el $50 \%$ de los alumnos extra regionales de la Región Metropolitana. Para el caso de la Quinta Región, la Metropolitana y la Sexta proveen el 54\% de los alumnos con AFI extrarregionales. Es de notar esta característica de la Quinta Región, que la ha proyectado en el nivel nacional como un importante centro universitario, generando y atrayendo buenos puntajes.

\subsubsection{Alumnos de tramos 4 y 5}

El subconjunto de alumnos ubicados en los tramos 4 y 5 del AFI son ponderados con 9 y 12 unidades de AFI, respectivamente. Los 11.000 alumnos en estos tramos concentran el $67 \%$ del aporte, que para el año 2002 significó 11.390 millones de pesos. Estos alumnos registraron un puntaje mayor o igual a 688 puntos en el proceso de admisión 2001. Como se aprecia en el cuadro $\mathrm{N}^{\circ} 3$, a medida que se aumenta de tramo, se marcan más claramente las características dominantes de los alumnos con AFI, es decir, mayor participación de alumnos de colegios privados, hombres, de la Región Metropolitana y egresados de la última promoción de enseñanza media.

\section{Cuadro $\mathrm{N}^{\circ} 3$}

Mayores participaciones según grupos alto y bajo de tramos de AFI

\begin{tabular}{|l|l|l|l|}
\hline Características & \multicolumn{1}{|c|}{ Tramo 1-3 } & \multicolumn{1}{c|}{ Tramo 4-5 } & \multicolumn{1}{c|}{ Total } \\
\hline Sexo & $52.5 \%$ hombres & $59.6 \%$ hombres & $55.3 \%$ hombres \\
\hline $\begin{array}{l}\text { Colegio } \\
\text { de procedencia }\end{array}$ & $\begin{array}{l}\text { Participación de colegios } \\
\text { particulares del orden del }\end{array}$ & $\begin{array}{l}55.5 \% \text { colegios } \\
\text { 30\%, con máximo de } \\
34.5 \%\end{array}$ & $\begin{array}{l}42.9 \% \text { colegios } \\
\text { particulares }\end{array}$ \\
\hline $\begin{array}{l}\text { Región } \\
\text { de procedencia }\end{array}$ & $\begin{array}{l}45.95 \% \text { Región } \\
\text { Metropolitana }\end{array}$ & $\begin{array}{l}58.7 \% \text { Región } \\
\text { Metropolitana }\end{array}$ & $\begin{array}{l}51.0 \% \text { Región } \\
\text { Metropolitana }\end{array}$ \\
\hline Promoción de egreso & $\begin{array}{l}51.9 \% \text { de la promoción } \\
\text { del año }\end{array}$ & $\begin{array}{l}65.6 \% \text { de la } \\
\text { promoción del año }\end{array}$ & $\begin{array}{l}\text { 60.3\% de la } \\
\text { promoción del año }\end{array}$ \\
\hline
\end{tabular}




\subsection{Resultados de la asignación}

\subsubsection{Variación del puntaje de corte}

El puntaje de corte de los 27.500 mejores puntajes se ha incrementado paulatinamente, debido al aumento de alumnos inscritos en la PAA y al mayor número de buenos puntajes que se matriculan. En 1996 bastaron 567.5 puntos para obtener AFI. En el año 2002 fueron necesarios 612 puntos como mínimo. Para el año 2003 proyectamos que el puntaje de corte estará alrededor de los 620 puntos (gráfico 7 y apéndice $\mathrm{N}^{\mathrm{o}} 6$ ).

En el año 1990 los 27.500 alumnos con AFI representaban el $29 \%$ de los que ingresaban a la educación superior. En el año 2001 esa cantidad representó sólo el 18\%. Para alcanzar participaciones cercanas al porcentaje del año 1990 sería necesario asignar AFI a los mejores 45.000 puntajes aproximadamente, pero ello llevaría al puntaje de corte a ubicarse cerca de los 550 puntos.

\section{Gráfico 7}

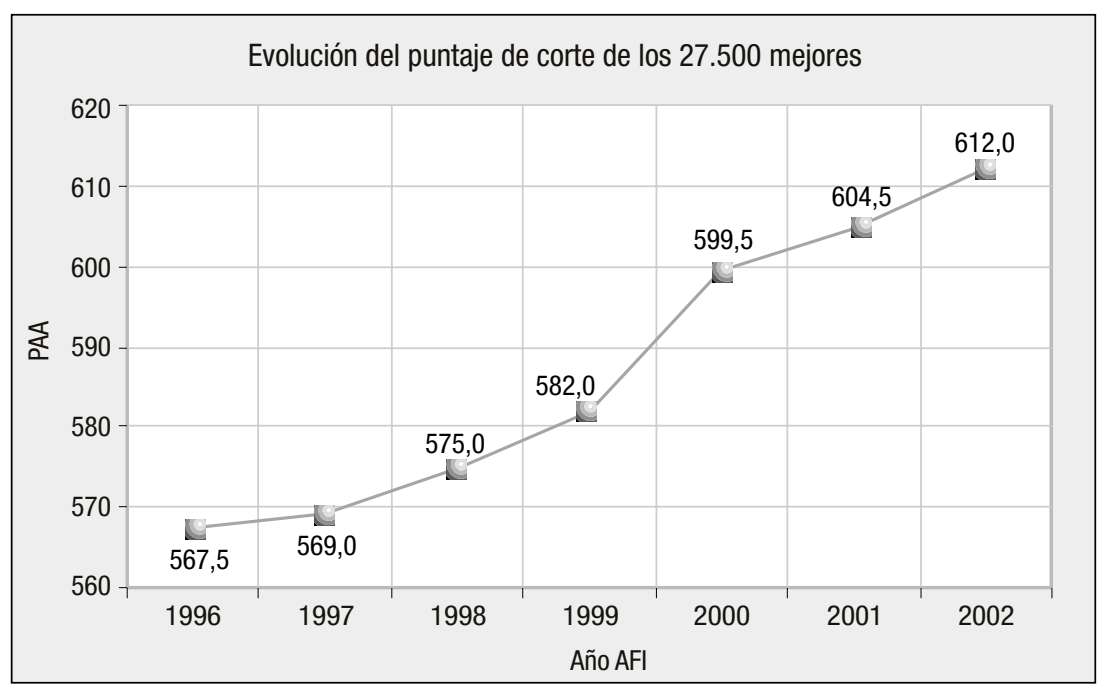




\subsubsection{Concentración del AFI en instituciones del Consejo de Rectores} Aunque las universidades del Consejo de Rectores aún concentran más del $81 \%$ de los alumnos con AFI y el $84 \%$ de los recursos, el despliegue de estrategias del sector privado para capturar a estos alumnos, conjuntamente con el mayor desarrollo y consolidación de las instituciones del sector, la obtención de la autonomía, y la apertura de carreras en el área de la salud, han resultado en un aumento en la participación de las universidades privadas en el AFI de un 15\% a $19 \%$ de los alumnos y de un $11.1 \%$ a un $15.5 \%$ en los recursos entre 1991 y 2002 (véase gráfico 8 y apéndice $\mathrm{N}^{\circ} 7$ ).

Gráfico 8

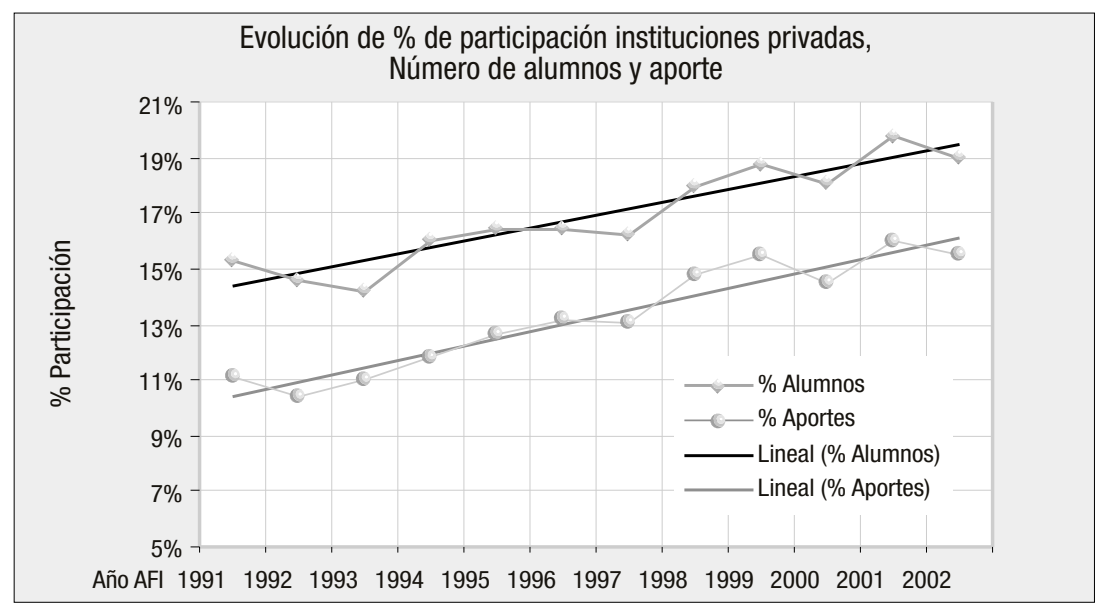

El número de alumnos por tramo de AFI del año 2002 muestra que las instituciones del Consejo de Rectores presentan una distribución que se concentra en los tramos altos, partiendo de un $22.1 \%$ en el tramo 5, hasta llegar a un $17.7 \%$ en el tramo 1. En las instituciones privadas se da la relación contraria, concentrándose sus alumnos en los tramos bajos, partiendo con un $25.9 \%$ en el tramo 1 , hasta llegar al $11.1 \%$ en el tramo 5.

No obstante, el crecimiento en la participación de las instituciones privadas entre 1991 y 2002 se ha dado en los tramos altos (5 y 4). Al mismo tiempo, las instituciones tradicionales han aumentado su participación en los tramos bajos (1 y 2). 
Gráfico 9

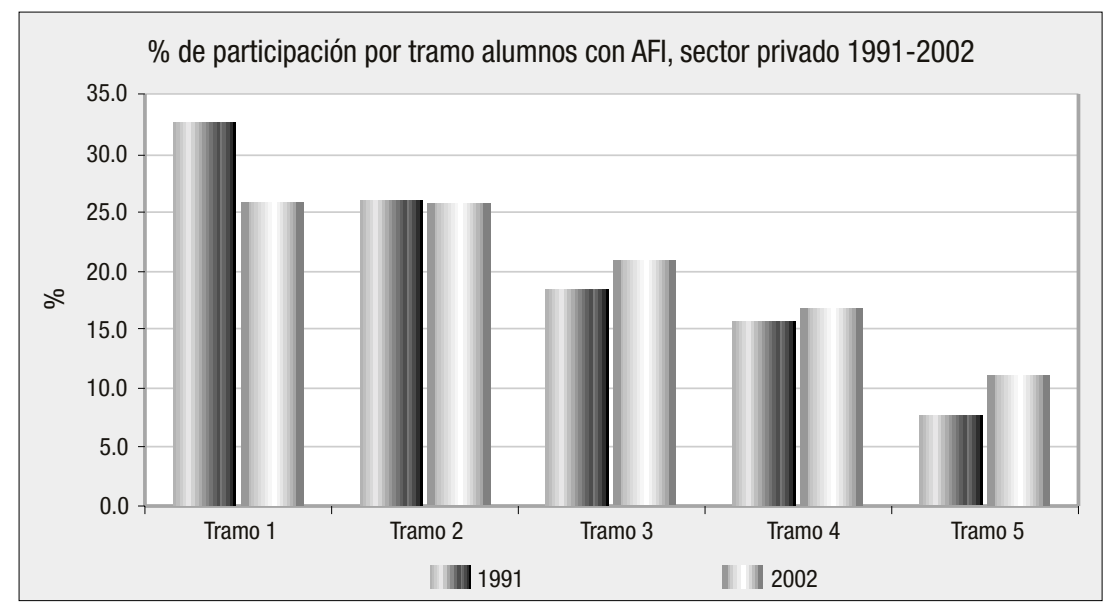

$\mathrm{Al}$ analizar el tramo 5, de los 5.500 alumnos con mejores puntajes, se advierte que las instituciones privadas han pasado de una participación del $5.71 \%$ a un $10.46 \%$. La Pontificia Universidad Católica de Chile (PUC) ha mantenido el liderazgo en este tramo desde 1997, año en que superó a la Universidad de Chile, la cual venía mostrando una caída sistemática hasta el año 1998, cuando estabilizó su participación en el orden del $27 \%$. Por su parte, la PUC, después de alcanzar su máximo el año 1998, con 30.6\%, cae hasta llegar a un 28\% el año 2002 (gráfico 10 y apéndices No 8 y 13).

\subsubsection{Concentración en universidades}

Las universidades concentran $99.2 \%$ de los recursos y el $98.5 \%$ de los alumnos con AFI. En 1991 los CFT captaban el 1.99\% de los alumnos con AFI y el $0.99 \%$ de los recursos; para el año 2002 estas cifras habían caído al $0.24 \%$ de los alumnos y el $0.13 \%$ de los recursos. Considerando que los CFT poseen el 19\% de la matrícula nueva del sistema, se advierte que para ellos el AFI carece de relevancia. Otro tanto sucede con los institutos profesionales(IP), que presentan hoy cifras de participación más bajas que las de los CFT en 1990 (apéndice No 9).

\subsubsection{Concentración regional}

Tres regiones acaparan el 86\% de los recursos del AFI: la Región Metropolitana con mucha distancia a la cabeza, con el 57\%; le siguen la Quinta con el 18\% y la Octava con el $11.7 \%$ (apéndice $\mathrm{N}^{\circ} 10$ ). 
Gráfico 10

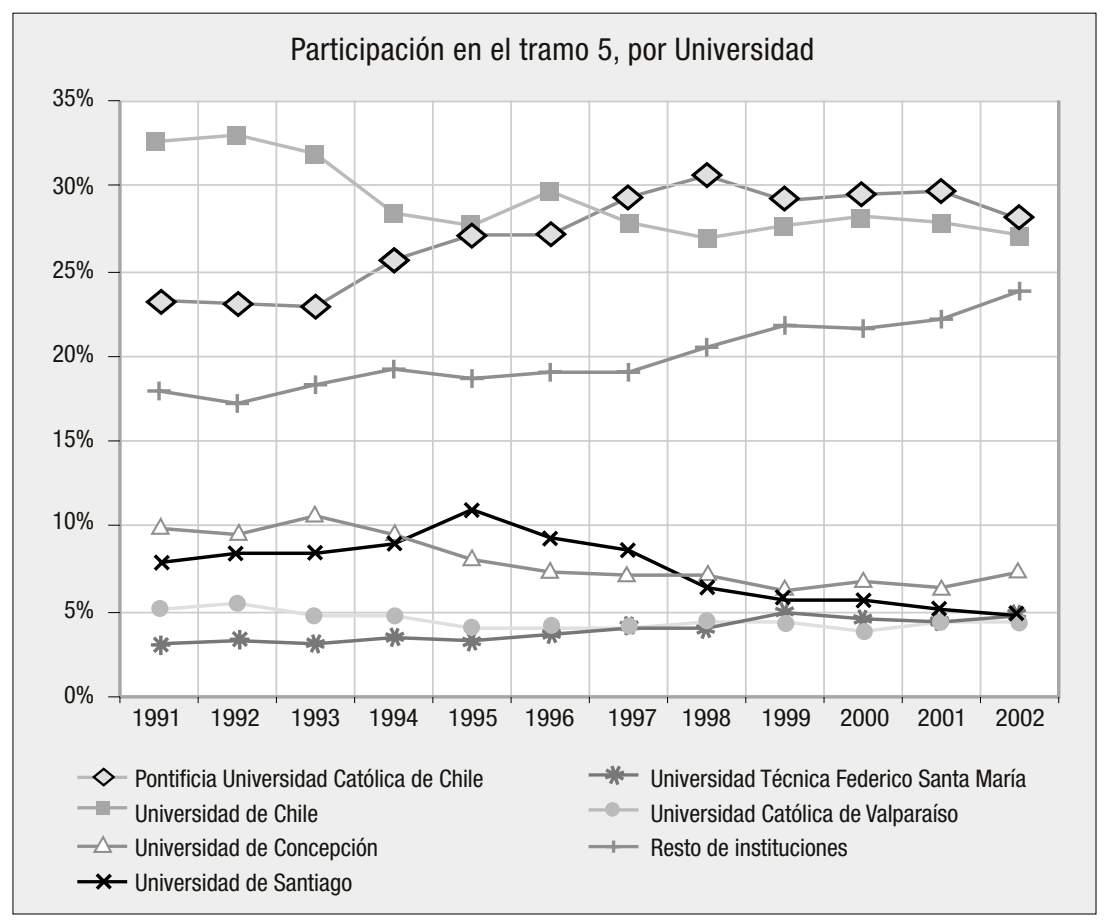

Como se dijo precedentemente, esta concentración de alumnos de buenos puntajes está relacionada con la capacidad de la región de generarlos, de retenerlos y de atraerlos desde otras regiones

\subsubsection{Concentración institucional}

El 80\% de los recursos del AFI benefician a sólo 13 universidades, y más del $35 \%$ de éste se reparte entre dos universidades solamente. Debido a este grado de concentración, las modificaciones al sistema tendrán su mayor impacto, negativo o positivo, sobre estas 13 universidades (apéndice $\mathrm{N}^{\circ} 11$ ).

Si se analiza la distribución de recursos al interior de las universidades privadas también se detecta una alta concentración: el $82 \%$ de los recursos beneficia a 9 universidades, y dos de ellas concentran más del 33\%. 
En el caso de los IP los pocos recursos que captan están aun más concentrados: el $83 \%$ de ellos en cuatro instituciones y más del $71 \%$ en sólo dos .

\subsubsection{Concentración en carreras}

El aporte también se concentra en determinadas carreras. Es así que más de $80 \%$ de los recursos están asignados al 16\% de las carreras. También existe una concentración en determinadas instituciones dentro de dichas carreras. Por ejemplo, para 1998, Ingeniería Comercial tenía el 9\% del total de los recursos AFI, pero dentro de esta carrera, más del $80 \%$ de los recursos fue a un $28 \%$ de las instituciones. Estos grados y formas de concentración sugieren una alta capacidad de discriminación de niveles de calidad entre los postulantes con altos puntajes: así como la calidad es escasa y no está repartida uniformemente entre las instituciones, del mismo modo el AFI no se encuentra repartido equilibradamente en el sistema, sino concentrado en pocas carreras e instituciones.

\section{MODIFICACIONES AL AFI}

De concretarse legalmente la sustitución de la PAA por otro mecanismo de selección para la educación superior, para mantener el AFI sería necesario modificar el artículo $3^{\circ}$ del DFL $\mathrm{N}^{\circ} 4$, que engancha el AFI con la PAA. Las declaraciones de algunas autoridades acerca de lo socialmente regresivo que sería el AFI hacen suponer que la intención del gobierno iría más allá de vincular al AFI con la nueva prueba, orientándose más bien a : a) incorporar las notas de enseñanza media como factor, lo cual estaría en sintonía con la filosofía del SIES de vincular más estrechamente el acceso a la Universidad con los resultados de la enseñanza media; $b$ ) incorporar variables socioeconómicas a la asignación del AFI; c) eliminarlo. Con todo, no existe una posición oficial por parte del Ministerio de Educación en esta materia; por su parte, existe una iniciativa parlamentaria, en el Congreso desde 1995, que propone incorporar las notas de enseñanza media en el puntaje considerado para la determinación del AFI.

Antes de introducir cualquier cambio, es importante que el gobierno proyecte los efectos que las modificaciones tendrían en el comportamiento de alumnos e instituciones, de modo de evitar resultados no deseados pero previsibles. 
Como una contribución a este ejercicio de evaluación ex ante, analizamos a continuación el efecto que tendría en el sistema la introducción de las notas de enseñanza media como factor generador de AFI, con una ponderación del 20\%, que parece ser una de las propuestas de cambios al AFI con más adeptos. Para ello se tomó a los alumnos con AFI el año $1998^{11}$ y se recalculó el AFI según el nuevo conjunto de indicadores. Los efectos serían los siguientes:

- Las mujeres aumentan su participación de un $42 \%$ del aporte a un $45 \%$, debido a que en promedio tienen mejores notas de enseñanza media que los hombres (apéndice $\mathrm{N}^{\mathrm{o}} 12$ ).

- Los alumnos de colegios particulares pagados bajarían levemente su participación, de un $50.1 \%$ a un $49.6 \%$ de los recursos, disminución que sería absorbida en su totalidad por los alumnos de colegios subvencionados.

- Las instituciones privadas, considerando universidades, institutos profesionales y centros de formación técnica bajarían su participación de un $15.4 \%$ a un $14.1 \%$.

- Como sector, las universidades del Consejo de Rectores son las únicas favorecidas con este cambio, mediante un aumento de su participación en los recursos, aunque algunas de dichas universidades bajarían sus aportes en forma significativa. En balance, un $80 \%$ de las universidades tradicionales subiría sus aportes respecto de lo que recibieron en 1998, mientras que sólo el $19 \%$ de las universidades privadas aumentaría los suyos. En tanto mayor sea la ponderación de las notas, menor será la proporción de universidades que resultan beneficiadas: con $50 \%$ notas y $50 \%$ PAA, las universidades beneficiadas bajan a $72 \%$ en el sector tradicional y $14 \%$ en el privado ${ }^{12}$.

11. Lo ideal habría sido trabajar con todos quienes dieron la PAA ese año, y no sólo con los que obtuvieron AFI, ya que con la introducción de las notas de enseñanza media como factor de asignación, la composición del grupo con AFI podría haber variado respecto del que lo obtuvo ese año. Esa base de datos no está disponible para nosotros. Sin embargo, la que presentamos es una aproximación bastante útil, porque de todas formas el puntaje PAA es el factor dominante ( $80 \%$ de ponderación), por lo cual la población beneficiada no debería cambiar radicalmente.

12. Para cada institución los resultados pueden variar dependiendo de la ponderación de las notas. Por ejemplo, a la USACH y la U. de Concepción les conviene una ponderación de notas del $20 \%$ tanto como una del $50 \%$. En cambio, a la U. de Chile y a la P. U. Católica de Chile les conviene un $20 \%$, pero no un $50 \%$ de ponderación de notas, porque este último porcentaje las pondría en un escenario menos ventajoso que el actual. (Cabe advertir que la estimación basada en un $50 \%$ de ponderación de las notas tiene un mayor grado de error, puesto que agregaría al AFI a un mayor número de alumnos no considerados en la muestra en estudio). 
- Las instituciones más favorecidas por este cambio serían las cuatro universidades que agrupan el $56.7 \%$ del aporte (U.de Chile, P. U. Católica de Chile, U. de Santiago y U. de Concepción), cuya participación crecería a un $58 \%$ de los recursos distribuidos por el AFI.

- Destacan la Universidad de Concepción, que presentaría el mayor crecimiento absoluto (65 millones de pesos), y la Universidad de Tarapacá, que presentaría el mayor crecimiento relativo (25\%).

- Entre las que registrarían una mayor caída bruta se encontrarían las universidades Tecnológica Metropolitana, la Central y la Diego Portales.

En suma, en esta simulación encontramos que la incorporación de las notas de enseñanza media como factor de asignación al AFI junto con la PAA beneficiaría a las postulantes mujeres y a las universidades del Consejo Rectores. También encontramos que no afectaría la actual composición socioeconómica de los alumnos receptores de AFI, medida por el tipo de colegio de procedencia del alumno.

\section{ALGUNAS IDEAS PARA MEJORAR EL AFI}

\subsection{Recapitulando lo que sabemos sobre la aplicación del AFI}

Antes de proponer mejoramientos al AFI conviene detenerse a recapitular los aspectos más destacables de nuestra evaluación de dicho instrumento. En primer lugar, tenemos que la distribución del AFI concuerda con la opinión de los expertos sobre la calidad de las instituciones de educación del país y sus ofertas de carreras. Esto no es de extrañar, puesto que el AFI se asigna con base en las preferencias estudiantiles y éstas, en buena medida, recogen el prestigio que tiene cada institución en la comunidad. Es irrelevante, para nuestros efectos, distinguir en qué medida es la distribución del AFI la que influye en la percepción de la gente sobre la calidad institucional, o en qué grado es esta percepción la que influye sobre los resultados del AFI a través de las decisiones de los postulantes. Lo cierto es que, en ausencia de un mecanismo como el AFI, no habría forma objetiva de medir la relativa preferencia de que gozan algunos programas y carreras sobre otros, entre los usuarios. 
Los montos de financiamiento asignados al AFI desde 1990 nos permiten colegir que los gobiernos de la Concertación han asumido sin mayor entusiasmo esta herencia de la reforma de la educación superior de 1981. De hecho, la relevancia estratégica del AFI como indicador de calidad, para las instituciones y para el público, excede con creces su importancia financiera, que alcanza hoy sólo el 7.6\% del presupuesto fiscal de educación superior. En un sistema de educación superior que se regula principalmente a través de la competencia, y donde existen asimetrías importantes de información, el AFI es una forma relativamente barata de inyectar al sistema información de alta calidad para la toma de decisiones. Esta función de orientación estratégica que cumple el AFI respecto de los actores del sistema se vería considerablemente mejorada, y por el mismo costo, si el MINEDUC manejara con más liberalidad los datos de la distribución del aporte, cuestión que probablemente, bajo la nueva legislación de probidad administrativa, se encuentra en todo caso obligado a hacer. Es indudable que el AFI ejerce sobre los requisitos de admisión una fuerza de gravedad hacia la PAA verbal y matemática por sobre otros criterios de selección. Nuestra simulación de la distribución que tendría un AFI que considerara las notas de enseñanza media sugiere que tal escenario concentraría aún más el AFI en las universidades tradicionales, y específicamente en algunas de ellas.

Por otra parte, al estar basado en la PAA, el AFI refleja los resultados socialmente desiguales de la prueba y de la educación secundaria. Sin embargo, incluir las notas en el AFI con una ponderación del $20 \%$, como algunos han propuesto, además de instar al recrudecimiento de la inflación de notas que ya se advierte en la secundaria, haría casi nada para corregir el sesgo del AFI hacia los alumnos egresados de colegios particulares pagados. La solución del problema de la equidad está en atacar sus causas en el sistema escolar, no sus síntomas en el acceso a la Universidad.

\subsection{Propuestas para mejorar el AFI}

\subsubsection{Incentivar la retención}

Con las actuales reglas un alumno puede recibir AFI como máximo dos veces, siempre que rinda la PAA nuevamente, obtenga un puntaje superior al corte de los 27.500 e ingrese a otra institución de educación superior. Este sistema premia dos veces por el mismo alumno, y premia 
al que se ha retirado ya una vez de sus estudios. Como un incentivo a la retención de los buenos alumnos, podría pensarse en asignar el aporte incluyendo sólo a los buenos puntajes que permanecen en la misma institución al año siguiente. La calidad de los alumnos hace poco probable que la institución los mantenga matriculados con independencia de sus méritos académicos. Por otra parte, la alta movilidad de estos alumnos, que pueden dar la PAA nuevamente sin temor de bajar sus puntajes, los convierte en confiables indicadores no sólo de la calidad que se supone tiene la institución -como hoy día-, sino del grado de satisfacción que experimentan como usuarios de ella luego de un año de conocerla.

Nuestro supuesto es que las instituciones que proyectan a la opinión pública más que lo que son pierden a sus buenos estudiantes que ven defraudadas sus expectativas. Las que, en cambio, cumplen lo que proyectan y prometen, los conservan. Asociar el AFI a estas últimas promovería la calidad real por sobre las imágenes de prestigio y solidez.

\subsubsection{Poner a disposición de las instituciones información más desagregada}

En atención a lo señalado en el punto 3.1.2, el MINEDUC debería poner a disposición de las instituciones los resultados de la distribución del AFI por tramo, carrera e institución, de modo de facilitar un análisis más detallado a dichas instituciones, y de permitir a los usuarios formarse juicios más precisos sobre la demanda por diversas carreras. Esta información permitiría también refinar los rankings de carreras, que están empezando a elaborar los medios de comunicación.

\subsubsection{Asignación por tipo de institución}

Si el gobierno está empeñado en promover el desarrollo de la educación técnica y de las opciones no universitarias, una compartimentación del AFI permitiría a los CFT y los IP competir entre ellos y no con universidades, como ocurre en la actualidad.

Podría, por ejemplo, dividir sobre la base de la matrícula de alumnos nuevos el fondo a distribuir. Así, por ejemplo, de los 17.000 millones que se asignan actualmente, quedarían 3.500 millones para los IP, 3.500 millones para los CFT y cerca de 10.000 millones para 
las universidades. Naturalmente, los requisitos para obtener AFI para los IP y los CFT tendrían que variar también respecto de los que hoy se aplican a las universidades, bajándose para ellos el puntaje de corte o estableciendo otros factores de mérito académico, además de la PAA.

\subsubsection{Estabilidad de las políticas de incentivos}

Como se indicó en el punto 3.1.1, en una política de incentivos es tan importante el volumen de recursos como su estabilidad. Los cambios en las conductas de las organizaciones requieren de estímulos sostenidos, de forma que los modos de acción deseados se vayan internalizando en las organizaciones y legitimando en la sociedad. Podría argumentarse que, tras veinte años de aplicación del AFI, la idea de competir por buenos estudiantes está ya institucionalizada en Chile, y que el incentivo monetario ya no es necesario para apoyarla. Sin embargo, no debe perderse de vista que las reglas del AFI sólo se han mantenido estables desde 1989. En segundo lugar, las universidades privadas han adquirido plena capacidad de competir sólo desde la obtención de la autonomía plena, condición de que goza un número importante de ellas desde hace dos o tres años (y que explica en parte el aumento de la participación de las universidades privadas en los tramos altos del AFI observado en los últimos años).

En otras palabras, el mercado de la educación superior en Chile se está consolidando recién en estos años, y el AFI es más necesario que nunca para contribuir a guiar su desarrollo hacia comportamientos institucionales que subrayen la orientación al usuario y a la calidad de servicios y productos, como factores clave en la competitividad de las instituciones de educación superior 


\section{Apéndice $\mathrm{N}^{\circ} 1$}

Universidades según ranking Qué Pasa,

$\%$ de alumnos de primer año con AFI

y número de UAFI por alumnos de primer año

\begin{tabular}{lll}
\hline Universidad & $\begin{array}{c}\text { Promedio nota } \\
\text { ranking }\end{array}$ \\
\hline
\end{tabular}

Católica de Chile

De Chile

6.54

6.33

T. Federico Santa María

5.84

De Concepción

Austral de Chile

UC. De Valparaíso

5.82

5.54

5.41

Adolfo Ibáñez

5.41

Diego Portales

De Talca

5.37

5.25

De los Andes

5.17

De la Frontera

Católica del Norte

4.97

4.97

Alberto Hurtado

4.93

Andrés Bello

4.84

Del Bío-Bío

De Valparaíso

Del Desarrollo

Mayor

4.80

4.67

4.66

4.64

Católica de Concepción

4.64

Finis Terrae

4.63

Católica del Maule

4.48

De La Serena

4.44

Central de Chile

4.42

$95.3 \%$
$88.2 \%$
$59.0 \%$
$61.8 \%$
$43.0 \%$
$77.6 \%$
$63.0 \%$
$42.5 \%$
$65.8 \%$
$61.9 \%$
$36.5 \%$
$33.2 \%$
$45.3 \%$
$14.2 \%$
$31.3 \%$
$56.4 \%$
$22.8 \%$
$34.1 \%$
$26.7 \%$
$41.4 \%$
$29.8 \%$
$26.4 \%$
$29.7 \%$

\section{Apéndice $\mathrm{N}^{\circ} 2$}

Alumnos por tramo, puntaje promedio PAA,

Puntaje promedio notas enseñanza media, por tipo dependencia del colegio, AFI 1998.

\begin{tabular}{|c|c|c|c|c|c|c|c|c|}
\hline \multirow[b]{2}{*}{ Dependencia } & \multirow{2}{*}{$\begin{array}{c}\text { Tramo } 1 \\
\text { Bajo }\end{array}$} & \multirow[b]{2}{*}{ Tramo 2} & \multirow[b]{2}{*}{ Tramo 3} & \multirow[b]{2}{*}{ Tramo 4} & \multirow{2}{*}{$\begin{array}{c}\text { Tramo } 5 \\
\text { Alto }\end{array}$} & \multirow[b]{2}{*}{ Total } & \multirow[b]{2}{*}{ PAA } & \multirow[b]{2}{*}{ Notas } \\
\hline & & & & & & & & \\
\hline Particular pagado & 1.607 & 1.866 & 2.230 & 2.742 & 3.377 & 11.822 & 670.5 & 606.0 \\
\hline \multicolumn{9}{|l|}{ Particular } \\
\hline subvencionado & 1.928 & 1.892 & 1.733 & 1.423 & 1.060 & 8.036 & 646.1 & 597.7 \\
\hline Municipal & 1.890 & 1.778 & 1.612 & 1.344 & 1.066 & 7.690 & 646.8 & 584.6 \\
\hline Sin información & 7 & 7 & 3 & 6 & 1 & 24 & & \\
\hline Total & 5.432 & 5.543 & 5.578 & 5.515 & 5.504 & 27.572 & 656.7 & 597.5 \\
\hline
\end{tabular}




\begin{tabular}{|lrcrc|}
\hline \multicolumn{5}{|c|}{ Apéndice N $\mathrm{N}^{0} 3$} \\
\multicolumn{4}{|c|}{$\begin{array}{l}\text { Alumnos con AFI, puntaje PAA (promedio verbal y matemáticas), } \\
\text { promedio notas enseñanza media, según sexo, datos AFI 1998. }\end{array}$} \\
\hline Sexo & Notas EM & PAA & Número & Participación \\
\hline Hombres & 576.0 & 660.5 & 15.259 & $55.3 \%$ \\
Mujeres & 624.2 & 652.0 & 12.313 & $44.7 \%$ \\
Total & 597.54 & 656.74 & 27.572 & $100.0 \%$ \\
\hline
\end{tabular}

\begin{tabular}{|c|c|c|c|c|c|c|c|}
\hline \multicolumn{8}{|c|}{$\begin{array}{c}\text { Apéndice } \mathrm{N}^{\mathbf{0}} 4 \\
\text { Número alumnos con AFI, } \\
\text { por tramo y año de egreso de enseñanza media, AFI } 1998\end{array}$} \\
\hline \multicolumn{3}{|c|}{ Tramo 1} & \multicolumn{5}{|c|}{ Tramo 5} \\
\hline Año & Bajo & Tramo 2 & Tramo 3 & Tramo 4 & Alto & Total & $\%$ \\
\hline Otros & 108 & 130 & 125 & 129 & 102 & 594 & $2.2 \%$ \\
\hline 90 & 42 & 43 & 43 & 56 & 33 & 217 & $0.8 \%$ \\
\hline 91 & 52 & 49 & 59 & 62 & 47 & 269 & $1.0 \%$ \\
\hline 92 & 91 & 90 & 84 & 100 & 69 & 434 & $1.6 \%$ \\
\hline 93 & 129 & 110 & 107 & 79 & 67 & 492 & $1.8 \%$ \\
\hline 94 & 187 & 190 & 181 & 143 & 152 & 853 & $3.1 \%$ \\
\hline 95 & 367 & 348 & 323 & 298 & 261 & 1.597 & $5.8 \%$ \\
\hline 96 & 1.466 & 1.414 & 1.403 & 1.191 & 1.005 & 6.479 & $23.5 \%$ \\
\hline 97 & 2.990 & 3.169 & 3.253 & 3.457 & 3.768 & 16.637 & $60.3 \%$ \\
\hline Total & 5.432 & 5.543 & 5.578 & 5.515 & 5.504 & 27.572 & \\
\hline
\end{tabular}




\begin{tabular}{|c|c|c|c|c|c|c|}
\hline \multicolumn{7}{|c|}{$\begin{array}{c}\text { Apéndice } \mathrm{N}^{\circ} 5 \\
\text { Número de alumnos AFI según región de proveniencia, } \\
\text { alumnos AFI que se matriculan en su región, total alumnos AFI matriculados } \\
\text { por región. AFI } 1998\end{array}$} \\
\hline Región & $\begin{array}{l}\text { Número de } \\
\text { alumnos } \\
\text { provenientes } \\
\text { de la región }\end{array}$ & 1 & $\begin{array}{l}\text { Alumnos de la } \\
\text { región que se } \\
\text { matriculan en } \\
\quad \text { ésta }\end{array}$ & $\begin{array}{l}\% \text { de } \\
\text { retención de } \\
\text { la región }\end{array}$ & $\begin{array}{l}\text { Total alumnos } \\
\text { matriculados en } \\
\text { la región }\end{array}$ & $\begin{array}{l}\text { Diferencia, } \\
\text { (alumnos de } \\
\text { otras regiones) }\end{array}$ \\
\hline 1 & 497 & $1.8 \%$ & 202 & $40.6 \%$ & 297 & 95 \\
\hline 2 & 790 & $2.9 \%$ & 525 & $66.5 \%$ & 953 & 428 \\
\hline 3 & 344 & $1.2 \%$ & 57 & $16.6 \%$ & 74 & 17 \\
\hline 4 & 785 & $2.8 \%$ & 323 & $41.1 \%$ & 507 & 184 \\
\hline 5 & 3.171 & $11.5 \%$ & 2.670 & $84.2 \%$ & 4.683 & 2.013 \\
\hline 6 & 1.131 & $4.1 \%$ & 0 & $0.0 \%$ & 0 & 0 \\
\hline 7 & 1.267 & $4.6 \%$ & 613 & $48.4 \%$ & 853 & 240 \\
\hline 8 & 3.040 & $11.0 \%$ & 2.471 & $81.3 \%$ & 3.225 & 754 \\
\hline 9 & 894 & $3.2 \%$ & 624 & $69.8 \%$ & 977 & 353 \\
\hline 10 & 1.075 & $3.9 \%$ & 552 & $51.3 \%$ & 855 & 303 \\
\hline 11 & 114 & $0.4 \%$ & 0 & $0.0 \%$ & 0 & 0 \\
\hline 12 & 326 & $1.2 \%$ & 86 & $26.4 \%$ & 93 & 7 \\
\hline Metropolitana & 14.060 & $51.0 \%$ & 12.842 & $91.3 \%$ & 15.055 & 2.213 \\
\hline Extranjero & 73 & $0.3 \%$ & & & 0 & \\
\hline Total & 27.572 & $100.0 \%$ & 20.965 & $76.0 \%$ & 27.572 & \\
\hline
\end{tabular}

\begin{tabular}{|cccccccc|}
\hline \multicolumn{7}{|c|}{ Apéndice N ${ }^{\circ}$ 6 } \\
\hline Tramo & 1996 & 1997 & 1998 & 1999 & 2000 & 2001 & 2002 \\
\hline 1 & 567.50 & 569.00 & 575.00 & 582.00 & 599.50 & 604.50 & 612.00 \\
\hline 2 & 593.50 & 597.00 & 603.00 & 608.00 & 624.00 & 627.00 & 634.00 \\
\hline 3 & 621.00 & 625.50 & 632.50 & 635.00 & 649.50 & 652.00 & 659.50 \\
\hline 4 & 651.00 & 657.00 & 664.00 & 665.50 & 687.50 & 680.50 & 688.00 \\
\hline 5 & 688.00 & 696.00 & 702.50 & 704.00 & 712.00 & 719.50 & 723.00 \\
\hline
\end{tabular}




\begin{tabular}{|c|c|c|c|c|}
\hline \multicolumn{5}{|c|}{$\begin{array}{c}\text { Apéndice } \mathrm{N}^{\mathrm{o}} 7 \\
\text { Evolución de las instituciones del } \\
\text { Consejo de Rectores e instituciones privadas, número y aporte (1991-2002) }\end{array}$} \\
\hline \multirow[b]{2}{*}{ Año AFI } & \multicolumn{2}{|c|}{$\begin{array}{c}\text { Instituciones Consejo } \\
\text { de Rectores }\end{array}$} & \multicolumn{2}{|c|}{ Instituciones privadas } \\
\hline & $\begin{array}{l}\text { Porcentaje de los } \\
\text { alumnos con AFI }\end{array}$ & $\begin{array}{c}\text { Porcentaje } \\
\text { de los aportes }\end{array}$ & $\begin{array}{l}\text { Porcentaje de los } \\
\text { alumnos con AFI }\end{array}$ & $\begin{array}{c}\text { Porcentaje } \\
\text { de los aportes }\end{array}$ \\
\hline 1991 & $84.7 \%$ & $88.9 \%$ & $15.3 \%$ & $11.1 \%$ \\
\hline 1992 & $85.4 \%$ & $89.6 \%$ & $14.6 \%$ & $10.4 \%$ \\
\hline 1993 & $85.8 \%$ & $89.0 \%$ & $14.2 \%$ & $11.0 \%$ \\
\hline 1994 & $84.0 \%$ & $88.2 \%$ & $16.0 \%$ & $11.8 \%$ \\
\hline 1995 & $83.5 \%$ & $87.4 \%$ & $16.5 \%$ & $12.6 \%$ \\
\hline 1996 & $83.5 \%$ & $86.9 \%$ & $16.5 \%$ & $13.1 \%$ \\
\hline 1997 & $83.8 \%$ & $86.9 \%$ & $16.2 \%$ & $13.1 \%$ \\
\hline 1998 & $82.0 \%$ & $85.2 \%$ & $18.0 \%$ & $14.8 \%$ \\
\hline 1999 & $81.2 \%$ & $84.5 \%$ & $18.8 \%$ & $15.5 \%$ \\
\hline 2000 & $82.0 \%$ & $85.5 \%$ & $18.0 \%$ & $14.5 \%$ \\
\hline 2001 & $80.2 \%$ & $84.0 \%$ & $19.8 \%$ & $16.0 \%$ \\
\hline 2002 & $81.0 \%$ & $84.5 \%$ & $19.0 \%$ & $15.5 \%$ \\
\hline
\end{tabular}

\begin{tabular}{|c|c|c|c|c|c|c|c|}
\hline \multicolumn{8}{|c|}{$\begin{array}{c}\text { Apéndice } \mathrm{N}^{\circ} 8 \\
\text { Instituciones con mayor participación en el tramo } 5 \\
\text { (5.500 mejores puntajes), 1990-2002 }\end{array}$} \\
\hline Año AFI & $\begin{array}{l}\text { Pontificia } \\
\text { Universidad } \\
\text { Católica de } \\
\text { Chile }\end{array}$ & $\begin{array}{l}\text { Universidad } \\
\text { de Chile }\end{array}$ & $\begin{array}{c}\text { Universidad } \\
\text { de } \\
\text { Concepción }\end{array}$ & $\begin{array}{l}\text { Universidad } \\
\text { de Santiago }\end{array}$ & $\begin{array}{l}\text { Universidad } \\
\text { Técnica } \\
\text { Federico } \\
\text { Santa María }\end{array}$ & $\begin{array}{l}\text { Universidad } \\
\text { Católica de } \\
\text { Valparaíso }\end{array}$ & $\begin{array}{c}\text { Resto } \\
\text { instituciones }\end{array}$ \\
\hline 1991 & $23.34 \%$ & $32.66 \%$ & $9.92 \%$ & $7.85 \%$ & $3.05 \%$ & $5.20 \%$ & $17.97 \%$ \\
\hline 1992 & $23.07 \%$ & $32.96 \%$ & $9.44 \%$ & $8.46 \%$ & $3.30 \%$ & $5.49 \%$ & $17.29 \%$ \\
\hline 1993 & $22.94 \%$ & $31.90 \%$ & $10.55 \%$ & $8.37 \%$ & $3.20 \%$ & $4.74 \%$ & $18.31 \%$ \\
\hline 1994 & $25.59 \%$ & $28.37 \%$ & $9.49 \%$ & $8.90 \%$ & $3.55 \%$ & $4.83 \%$ & $19.27 \%$ \\
\hline 1995 & $27.21 \%$ & $27.63 \%$ & $8.13 \%$ & $10.95 \%$ & $3.29 \%$ & $4.10 \%$ & $18.69 \%$ \\
\hline 1996 & $27.03 \%$ & $29.63 \%$ & $7.27 \%$ & $9.42 \%$ & $3.64 \%$ & $3.96 \%$ & $19.05 \%$ \\
\hline 1997 & $29.32 \%$ & $27.87 \%$ & $7.08 \%$ & $8.58 \%$ & $4.04 \%$ & $4.00 \%$ & $19.12 \%$ \\
\hline 1998 & $30.59 \%$ & $26.88 \%$ & $7.11 \%$ & $6.45 \%$ & $4.01 \%$ & $4.36 \%$ & $20.61 \%$ \\
\hline 1999 & $29.22 \%$ & $27.71 \%$ & $6.25 \%$ & $5.74 \%$ & $4.91 \%$ & $4.36 \%$ & $21.82 \%$ \\
\hline 2000 & $29.50 \%$ & $28.13 \%$ & $6.71 \%$ & $5.68 \%$ & $4.60 \%$ & $3.80 \%$ & $21.58 \%$ \\
\hline 2001 & $29.70 \%$ & $27.81 \%$ & $6.44 \%$ & $5.19 \%$ & $4.34 \%$ & $4.34 \%$ & $22.19 \%$ \\
\hline 2002 & $28.00 \%$ & $27.19 \%$ & $7.31 \%$ & $4.69 \%$ & $4.69 \%$ & $4.33 \%$ & $23.80 \%$ \\
\hline
\end{tabular}




\begin{tabular}{|c|c|c|c|c|c|c|}
\hline \multicolumn{7}{|c|}{$\begin{array}{c}\text { Apéndice } \mathrm{N}^{\circ} 9 \\
\text { Evolución de la participación, por tipo de institución, } \\
\text { número de alumnos y aporte }\end{array}$} \\
\hline \multirow[b]{2}{*}{ Año AFI } & \multicolumn{3}{|c|}{ Número de alumnos con AFI } & \multicolumn{3}{|c|}{ Monto del aporte } \\
\hline & CFT & IP & Universidad & CFT & IP & Universidad \\
\hline 1991 & $1.99 \%$ & $7.11 \%$ & $90.90 \%$ & $0.99 \%$ & $4.77 \%$ & $94.23 \%$ \\
\hline 1992 & $1.53 \%$ & $5.90 \%$ & $92.57 \%$ & $0.71 \%$ & $4.06 \%$ & $95.23 \%$ \\
\hline 1993 & $1.10 \%$ & $5.67 \%$ & $93.23 \%$ & $0.56 \%$ & $3.76 \%$ & $95.68 \%$ \\
\hline 1994 & $1.34 \%$ & $1.92 \%$ & $96.74 \%$ & $0.59 \%$ & $1.01 \%$ & $98.40 \%$ \\
\hline 1995 & $1.09 \%$ & $1.89 \%$ & $97.03 \%$ & $0.48 \%$ & $1.02 \%$ & $98.50 \%$ \\
\hline 1996 & $0.69 \%$ & $1.61 \%$ & $97.70 \%$ & $0.35 \%$ & $0.89 \%$ & $98.77 \%$ \\
\hline 1997 & $0.50 \%$ & $1.63 \%$ & $97.86 \%$ & $0.26 \%$ & $0.81 \%$ & $98.93 \%$ \\
\hline 1998 & $0.43 \%$ & $1.83 \%$ & $97.74 \%$ & $0.22 \%$ & $0.97 \%$ & $98.82 \%$ \\
\hline 1999 & $0.37 \%$ & $1.79 \%$ & $97.84 \%$ & $0.18 \%$ & $0.89 \%$ & $98.93 \%$ \\
\hline 2000 & $0.29 \%$ & $1.24 \%$ & $98.47 \%$ & $0.14 \%$ & $0.62 \%$ & $99.24 \%$ \\
\hline 2001 & $0.21 \%$ & $1.32 \%$ & $98.46 \%$ & $0.12 \%$ & $0.80 \%$ & $99.08 \%$ \\
\hline 2002 & $0.24 \%$ & $1.19 \%$ & $98.56 \%$ & $0.13 \%$ & $0.63 \%$ & $99.24 \%$ \\
\hline
\end{tabular}

\begin{tabular}{|c|c|c|c|c|c|c|c|c|c|c|c|c|c|c|}
\hline \multicolumn{15}{|c|}{$\begin{array}{l}\text { Apéndice No } 10 \\
\text { Participación en los recursos AFI, por región, años 1991-2002 }\end{array}$} \\
\hline \multicolumn{15}{|c|}{ Región } \\
\hline Año AFl & l & 2 & 3 & 4 & 5 & 6 & 7 & 8 & 9 & 10 & 12 & 13 & Sin info & Total \\
\hline 1991 & $1.3 \%$ & $2.9 \%$ & $0.2 \%$ & $1.0 \%$ & $16.6 \%$ & $0.0 \%$ & $1.5 \%$ & $13.6 \%$ & $2.4 \%$ & $4.2 \%$ & $0.3 \%$ & $54.0 \%$ & $2.0 \%$ & $100.0 \%$ \\
\hline 1992 & $1.4 \%$ & $2.7 \%$ & $0.2 \%$ & $1.1 \%$ & $15.9 \%$ & $0.0 \%$ & $1.7 \%$ & $13.9 \%$ & $2.5 \%$ & $3.6 \%$ & $0.2 \%$ & $55.2 \%$ & $1.5 \%$ & $100.0 \%$ \\
\hline 1993 & $0.8 \%$ & $2.4 \%$ & $0.2 \%$ & $1.0 \%$ & $15.2 \%$ & $0.0 \%$ & $2.3 \%$ & $13.7 \%$ & $3.0 \%$ & $3.5 \%$ & $0.1 \%$ & $56.7 \%$ & $1.2 \%$ & $100.0 \%$ \\
\hline 1994 & $0.9 \%$ & $3.3 \%$ & $0.2 \%$ & $1.3 \%$ & $15.1 \%$ & $0.0 \%$ & $2.3 \%$ & $12.8 \%$ & $3.2 \%$ & $3.4 \%$ & $0.2 \%$ & $56.0 \%$ & $1.3 \%$ & $100.0 \%$ \\
\hline 1995 & $0.9 \%$ & $2.8 \%$ & $0.1 \%$ & $1.5 \%$ & $13.2 \%$ & $0.0 \%$ & $2.5 \%$ & $11.8 \%$ & $3.3 \%$ & $3.3 \%$ & $0.2 \%$ & $59.1 \%$ & $1.2 \%$ & $100.0 \%$ \\
\hline 1996 & $0.7 \%$ & $2.8 \%$ & $0.2 \%$ & $1.3 \%$ & $14.1 \%$ & $0.0 \%$ & $2.2 \%$ & $11.5 \%$ & $2.9 \%$ & $2.7 \%$ & $0.1 \%$ & $60.6 \%$ & $0.9 \%$ & $100.0 \%$ \\
\hline 1997 & $0.6 \%$ & $3.0 \%$ & $0.3 \%$ & $1.3 \%$ & $15.0 \%$ & $0.0 \%$ & $2.4 \%$ & $10.7 \%$ & $2.9 \%$ & $2.7 \%$ & $0.2 \%$ & $60.1 \%$ & $0.8 \%$ & $100.0 \%$ \\
\hline 1998 & $0.7 \%$ & $2.9 \%$ & $0.2 \%$ & $1.2 \%$ & $15.5 \%$ & $0.0 \%$ & $2.3 \%$ & $10.9 \%$ & $3.1 \%$ & $2.6 \%$ & $0.2 \%$ & $59.5 \%$ & $0.8 \%$ & $100.0 \%$ \\
\hline 1999 & $0.6 \%$ & $2.8 \%$ & $0.2 \%$ & $1.1 \%$ & $16.3 \%$ & $0.0 \%$ & $2.4 \%$ & $10.2 \%$ & $2.8 \%$ & $2.3 \%$ & $0.2 \%$ & $60.5 \%$ & $0.7 \%$ & $100.0 \%$ \\
\hline 2000 & $0.6 \%$ & $2.5 \%$ & $0.2 \%$ & $1.1 \%$ & $16.5 \%$ & $0.0 \%$ & $2.5 \%$ & $11.0 \%$ & $2.7 \%$ & $2.5 \%$ & $0.2 \%$ & $59.7 \%$ & $0.5 \%$ & $100.0 \%$ \\
\hline 2001 & $0.7 \%$ & $2.5 \%$ & $0.1 \%$ & $1.0 \%$ & $16.2 \%$ & $0.0 \%$ & $2.7 \%$ & $10.8 \%$ & $2.5 \%$ & $2.4 \%$ & $0.1 \%$ & $60.6 \%$ & $0.5 \%$ & $100.0 \%$ \\
\hline 2002 & $0.6 \%$ & $2.5 \%$ & $0.2 \%$ & $1.0 \%$ & $18.0 \%$ & $0.0 \%$ & $3.2 \%$ & $11.7 \%$ & $2.6 \%$ & $2.4 \%$ & $0.1 \%$ & $57.2 \%$ & $0.4 \%$ & $100.0 \%$ \\
\hline
\end{tabular}




\begin{tabular}{|c|c|c|}
\hline \multicolumn{3}{|c|}{$\begin{array}{l}\text { Apéndice } \mathrm{N}^{\mathrm{o}} 11 \\
\text { Instituciones con mayor participación en AFI 2002, por sector. } \\
\text { Total, universidades privadas, institutos profesionales }\end{array}$} \\
\hline Todas las instituciones & $\begin{array}{l}\text { Participación } \\
\text { sector }\end{array}$ & $\begin{array}{l}\text { Participación } \\
\text { acumulada } \\
\text { sector }\end{array}$ \\
\hline UNIVERSIDAD DE CHILE & $18.9 \%$ & $18.9 \%$ \\
\hline PONTIFICIA UNIVERSIDAD CATÓLICA DE CHILE & $16.6 \%$ & $35.5 \%$ \\
\hline UNIVERSIDAD DE CONCEPCIÓN & $7.7 \%$ & $43.2 \%$ \\
\hline UNIVERSIDAD DE SANTIAGO & $7.4 \%$ & $50.6 \%$ \\
\hline UNIVERSIDAD CATÓLICA DE VALPARAÍSO & $6.7 \%$ & $57.4 \%$ \\
\hline UNIVERSIDAD TÉCNICA FEDERICO SANTA MARÍA & $4.9 \%$ & $62.3 \%$ \\
\hline UNIVERSIDAD DE VALPARAÍSO & $3.4 \%$ & $65.6 \%$ \\
\hline UNIVERSIDAD MAYOR & $3.1 \%$ & $68.8 \%$ \\
\hline UNIVERSIDAD TECNOLÓGICA METROPOLITANA & $2.5 \%$ & $71.3 \%$ \\
\hline UNIVERSIDAD AUSTRAL DE CHILE & $2.3 \%$ & $73.6 \%$ \\
\hline UNIVERSIDAD DE TALCA & $2.3 \%$ & $75.8 \%$ \\
\hline UNIVERSIDAD DE LA FRONTERA & $2.2 \%$ & $78.0 \%$ \\
\hline UNIVERSIDAD ADOLFO IBÁÑEZ & $2.1 \%$ & $80.2 \%$ \\
\hline \multicolumn{3}{|l|}{ Sólo universidades privadas } \\
\hline UNIVERSIDAD MAYOR & $20.16 \%$ & $20.16 \%$ \\
\hline UNIVERSIDAD ADOLFO IBÁÑEZ & $13.74 \%$ & $33.90 \%$ \\
\hline UNIVERSIDAD DE LOS ANDES & $9.14 \%$ & $43.05 \%$ \\
\hline UNIVERSIDAD DIEGO PORTALES & $8.62 \%$ & $51.67 \%$ \\
\hline UNIVERSIDAD NACIONAL ANDRÉS BELLO & $8.57 \%$ & $60.24 \%$ \\
\hline UNIVERSIDAD DEL DESARROLLO & $7.81 \%$ & $68.05 \%$ \\
\hline UNIVERSIDAD CENTRAL & $6.21 \%$ & $74.26 \%$ \\
\hline UNIVERSIDAD SAN SEBASTIÁN & $4.21 \%$ & $78.47 \%$ \\
\hline UNIVERSIDAD FINIS TERRAE & $3.87 \%$ & $82.34 \%$ \\
\hline \multicolumn{3}{|l|}{ Sólo institutos profesionales } \\
\hline INSTITUTO PROFESIONAL DUOC UC & $42.63 \%$ & $42.63 \%$ \\
\hline INSTITUTO PROFESIONAL INACAP & $28.55 \%$ & $71.18 \%$ \\
\hline INSTITUTO PROFESIONAL CAMPUS & $8.29 \%$ & $79.47 \%$ \\
\hline INSTITUTO PROFESIONAL ESCUELA MODERNA DE MÚSICA & $3.59 \%$ & $83.06 \%$ \\
\hline
\end{tabular}




\begin{tabular}{|lrrrc|}
\hline \multicolumn{5}{|c|}{$\begin{array}{c}\text { Apéndice } \mathrm{N}^{0} 12 \\
\text { Puntajes promedio PAA, notas EM, ponderado al 20\% notas EM, } \\
\text { según sexo alumnos AFI 1998 }\end{array}$} \\
\hline Sexo & Número & PAA & Notas EM & $\begin{array}{r}\text { Ponderado } \\
20 \% \text { notas }\end{array}$ \\
\hline Hombres & 15,259 & 660.6 & 576.0 & 643.7 \\
Mujeres & 12,313 & 652.0 & 624.2 & 646.4 \\
Total & 27,572 & 656.7 & 597.5 & 644.9 \\
Diferencia entre hombres y mujeres & 2,946 & 8.5 & -48.1 & -2.8 \\
\hline
\end{tabular}

\begin{tabular}{|c|c|c|c|c|c|c|}
\hline & Particip & $\begin{array}{r}\mathrm{A} \\
\text { ación en el t } \\
\text { por tipo ys }\end{array}$ & $\begin{array}{l}\text { péndice } \mathrm{N}^{\circ} \\
\text { amo } 5(5.5 \\
\text { ector de las }\end{array}$ & $\begin{array}{l}13 \\
00 \text { mejores } 1 \\
\text { institucione }\end{array}$ & puntajes), & \\
\hline Año AFI & Tipo & & & & & Total \\
\hline & CFT & IP & & Universidad & & general \\
\hline & Sin aporte & Con aporte & Sin aporte & Con aporte & Sin aporte & \\
\hline 1991 & $0.34 \%$ & $0.83 \%$ & $0.42 \%$ & $93.45 \%$ & $4.95 \%$ & $100.00 \%$ \\
\hline 1992 & $0.04 \%$ & $1.03 \%$ & $0.31 \%$ & $94.40 \%$ & $4.22 \%$ & $100.00 \%$ \\
\hline 1993 & $0.20 \%$ & $0.96 \%$ & $0.27 \%$ & $92.72 \%$ & $5.86 \%$ & $100.00 \%$ \\
\hline 1994 & $0.11 \%$ & & $0.16 \%$ & $93.88 \%$ & $5.85 \%$ & $100.00 \%$ \\
\hline 1995 & $0.11 \%$ & & $0.28 \%$ & $93.44 \%$ & $6.17 \%$ & $100.00 \%$ \\
\hline 1996 & $0.02 \%$ & & $0.45 \%$ & $92.42 \%$ & $7.11 \%$ & $100.00 \%$ \\
\hline 1997 & $0.11 \%$ & & $0.16 \%$ & $92.44 \%$ & $7.29 \%$ & $100.00 \%$ \\
\hline 1998 & $0.09 \%$ & & $0.20 \%$ & $91.69 \%$ & $8.01 \%$ & $100.00 \%$ \\
\hline 1999 & $0.02 \%$ & & $0.18 \%$ & $90.48 \%$ & $9.32 \%$ & $100.00 \%$ \\
\hline 2000 & $0.02 \%$ & & $0.16 \%$ & $91.09 \%$ & $8.73 \%$ & $100.00 \%$ \\
\hline 2001 & & & $0.22 \%$ & $90.24 \%$ & $9.54 \%$ & $100.00 \%$ \\
\hline 2002 & $0.02 \%$ & & $0.20 \%$ & $89.54 \%$ & $10.24 \%$ & $100.00 \%$ \\
\hline
\end{tabular}




\author{
DFL-4 \\ Fecha publicación: 20.01.1981 \\ Fecha promulgación: 14.01.1981 \\ Organismo: MINISTERIO DE EDUCACIÓN PÚBLICA \\ Última modificación: LEY N 18.768 \\ Fecha última modificacion: 29.12.1988 \\ Estado: ACTUALIZADO
}

\title{
Artículo $3^{\circ}$
}

Sin perjuicio del aporte referido en el artículo anterior, el Estado otorgará a todas las universidades, institutos profesionales y centros de formación técnica, reconocidos por aquél como instituciones de educación superior, anualmente un aporte fiscal indirecto, el que será distribuido de la siguiente forma:

1. El Ministerio de Educación Pública elaborará un listado con los primeros 27.500 puntajes de los alumnos matriculados en el primer año de estudios, en el año inmediatamente anterior, en las instituciones de educación superior, ordenado de menor a mayor de acuerdo con los puntajes obtenidos en la Prueba de Aptitud Académica, partes verbal y matemática.

2. Dicho listado será dividido en cinco tramos de similar número de alumnos cada uno, con factores de ponderación 1, 3, 6, 9 y 12 , respectivamente, para los tramos 1, 2, 3, 4 y 5 .

El tramo 1 corresponderá a los puntajes más bajos y el tramo 5 a los puntajes más altos. Los alumnos que hayan obtenido igual puntaje en la Prueba de Aptitud Académica deberán figurar en un mismo tramo. Al efecto se aumentará el número de alumnos del tramo en que figure la mayor cantidad del mismo puntaje y se disminuirá en la misma cantidad el otro tramo.

3. El número de alumnos de cada tramo será multiplicado por los factores correspondientes, establecidos en el punto anterior.

4. El monto base de recursos que se entregarán por cada alumno se determinará dividiendo la cantidad asignada para estos efectos en la Ley de Presupuestos por la suma del producto de las multiplicaciones obtenidas en el punto anterior. 
5. A dicho monto base se le aplicará el factor de ponderación que corresponda a cada alumno, según sea el tramo en que se ubique de acuerdo con su puntaje en la Prueba de Aptitud Académica, determinándose de esta manera el monto de recursos que se asignarán por cada alumno ubicado en los tramos 1 al 5 .

6. Para determinar el monto de aporte fiscal que por este concepto corresponde a cada institución de educación superior, se procederá de la siguiente forma:

a) Los alumnos matriculados en el año inmediatamente anterior en el primer año de estudios de cada institución de educación superior, se ubicarán en el tramo que les corresponda, de acuerdo con el puntaje obtenido en la Prueba de Aptitud Académica.

b) El número de alumnos que de esta forma resulte en cada tramo, se multiplicará por el monto de recursos determinado por cada tramo en el punto 5 .

c) La suma de los valores así obtenidos determinará el monto total para el año respectivo, que corresponderá a cada institución de educación superior.

7. En el listado de alumnos señalado en el número 1, no se considerarán los alumnos que ingresen por segunda vez a una misma institución de educación superior. Tampoco se considerarán los alumnos que ingresen por tercera vez, a una misma o a otra institución de educación superior.

8. El monto determinado para cada institución, será sancionado por decreto supremo expedido por intermedio del Ministerio de Educación Pública, el que deberá ser suscrito también por el Ministro de Hacienda, y se entregará mensualmente a cada una de ellas un duodécimo de dicha cantidad. 